\title{
Quasi-Stationary Distributions for Models of Heterogeneous Catalysis
}

\author{
Marcelo M. de Oliveira* and Ronald Dickman ${ }^{\dagger}$ \\ Departamento de Física, Instituto de Ciências Exatas, \\ Universidade Federal de Minas Gerais \\ C. P. 702, 30123-970, Belo Horizonte, MG - Brazil
}

(Dated: September 3, 2018)

\begin{abstract}
We construct the quasi-stationary (QS) distribution for two models of heterogeneous catalysis having two absorbing states: the ZGB model for the oxidation of $\mathrm{CO}$, and a version with noninstantaneous reactions. Using a mean-field-like approximation, we study the quasi-stationary surface coverages, moment ratios and the lifetime of the QS state. We also derive an improved, consistent one-site mean-field theory for the ZGB model.
\end{abstract}

PACS numbers: 82.20.-w,82.65.Jv,05.10.Gg,02.50.Ga,05.70.Ln

* mancebo@fisica.ufmg.br

† dickman@fisica.ufmg.br 


\section{INTRODUCTION}

The Ziff-Gullari-Barshard(ZGB) model [1] for the oxidation of carbon monoxide (CO) on a catalytic surface exhibits phase transitions between an active steady state and absorbing or "poisoned" states, in which the surface is saturated either by oxygen (O) or by CO. This model and its variants have stimulated much interest; many studies of the ZGB model have been published using deterministic mean-field equations [2, 3, 4] and Monte Carlo simulations. For reviews see [5, 6, 7].) In the present work we analyze quasi-stationary properties of the model. In the process, we revisit the one-site mean-field theory and formulate an improved version thereof.

The surface reactions described by the ZGB and allied models follow the LangmuirHinshelwood mechanism [8],

$$
\begin{array}{r}
\mathrm{CO}_{g a s}+v \rightarrow \mathrm{CO}_{a d s} \\
\mathrm{O}_{2 g a s}+2 v \rightarrow 2 \mathrm{O}_{a d s} \\
\mathrm{CO}_{a d s}+\mathrm{O}_{a d s} \rightarrow \mathrm{CO}_{2}+2 v,
\end{array}
$$

where $v$ denotes a vacant lattice site, and the subscripts indicate the state (gaseous or adsorbed) of the molecule. The product $\mathrm{CO}_{2}$ is understood to desorb immediately on formation. $\mathrm{CO}_{\text {gas }}$ molecules arrive at a rate $Y$ per site while $\mathrm{O}_{2}$ arrives at rate $(1-Y) \equiv \bar{Y}$.

Monte Carlo simulations 1] reveal that the model exhibits two phase transitions. For $Y<$ $y_{1}=0.391$ the lattice eventually poisons with $\mathrm{O}$ while for $Y>y_{2}=0.5256$ it poisons with CO. For $y_{1}<Y<y_{2}$ the system attains a reactive steady state. The first transition is found to be continuous while the second is strongly discontinuous. Mean-field-like descriptions based on cluster approximations 2] reproduce qualitatively the behavior of the system in the thermodynamic limit.

Large but finite systems appear to be stationary on any reasonable time scale (away from the phase boundaries), but eventually end up in one of the absorbing states (O-poisoned or CO-poisoned), via a fluctuation with a very small, but nonzero probability. Mean-field theories ignore such fluctuations, and so are incapable of treating finite systems. Since simulations and other numerical methods usually study finite systems, it is useful to develop approximate theoretical descriptions that account for finite system size. Experimental study of nanoscale catalytic systems is also of current interest [9]. The notion of a quasi-stationary 
(QS) distribution has proved to be a powerful tool in this context. For such models, the QS distribution describes the asymptotic (long-time) properties of a finite system conditioned on survival [10, 11, 12]. The quasi-stationary properties converge to the stationary properties when the system size $N \rightarrow \infty$.

In Ref. [13] QS distributions (QSD) are derived for various continuous-time Markov processes, e.g., the contact process and the Malthus-Verhulst process. In Ref. [14] the QSD for a discrete-time process is derived, using the Domany-Kinzel cellular automaton as an example. In all of these studies, mean-field-like approximations are used to find the QSD. Previous studies have focused on continuous phase transitions to an absorbing state, with the exception of Schlögl's second model [15], known to exhibit a discontinuous transition in mean field approximation, which was shown in Ref. 13] to exhibit a bimodal QSD at the transition. Such a bimodal distribution (e.g., of the order parameter) is a hallmark of a discontinuous phase transition. The phase transition in Schlögl's second model, with nearest-neighbor interactions, is however known to be continuous in $d<4$ dimensions [16]. Hence, aside from the particular interest of the ZGB model in the context of catalysis, the present work presents a QS analysis of a model known to exhibit a discontinuous transition.

The balance of this paper is organized as follows. In Sec. II, we briefly review the meanfield theory for the ZGB model at the site level, introducing modifications that take into account the prohibition against O-CO nearest-neighbor (NN) pairs on the surface. In Sec. III, we define the QS probability distribution and show how to derive it in an efficient way. This method is used to generate the results reported in Sec. IV; results for the case of noninstantaneous reactions (NI model) are also presented. Sec. V contains a summary of our results. The Appendix treats the ZGB model on a complete graph.

\section{SITE MEAN-FIELD APPROXIMATIONS}

The simplest mean-field description of the ZGB model is the site approximation. By assuming spatial homogeneity and neglecting all correlations between the occupancies of different sites, one obtains a closed description in terms of the $\mathrm{CO}$ and $\mathrm{O}$ coverages, i.e., the fraction of sites occupied by $\mathrm{CO}(\mathrm{O})$. To simplify the notation, from this point we will denote a $\mathrm{CO}$ molecule by $c$, a vacant site by $v$, and an $\mathrm{O}$ atom by $o$. The coverages will be denoted by $\theta_{i}$, for $i=c, v$ or $o$, with the normalization $\theta_{c}+\theta_{o}+\theta_{v}=1$. 
In the ZGB model reactions between adsorbed $c$ and $o$ are instantaneous, so that there is a strict prohibition against $c-o$ NN pairs. Previous formulations of the site approximation [2, 3, 4] effectively ignored this prohibition, leading to the appearance of an absorbing state in which a small fraction (about $0.2 \%$ ) of the sites are occupied by $o$, while the rest bear $c$ molecules. Elimination of this nonphysical effect is one motivation for reformulating the approximation; providing a systematic basis for the QS analysis is another. In the absence of any prohibitions, the site approximation treats each site as statistically independent, so that the joint probability $\theta_{i j}$ for a NN pair of sites to be in states $i$ and $j$ is $\theta_{i} \theta_{j}$. Our problem is to formulate an approximation that treats sites as independent, except for the fact that $\theta_{o c}=\theta_{c o}=0$. (We use $\theta_{i j}$ to denote the joint probability that, on the square lattice, the leftmost or lower site of the pair is in state $i$ and the other site in state $j$. By symmetry $\left.\theta_{j i}=\theta_{i j}.\right)$

On a square lattice of $N$ sites (with periodic boundaries, so that each site possesses exactly four NNs), there are $2 N$ NN pairs. Let $N_{i}$ be the number of sites in state $i$ and $N_{i j}$ the number of NN pairs with states $i$ and $j$, using the same convention as for $\theta_{i j}$. Then we have the relations

$$
\begin{gathered}
N_{v}=\frac{1}{2}\left(N_{v v}+N_{v o}+N_{v c}\right) \\
N_{o}=\frac{1}{2}\left(N_{o o}+N_{v o}\right)
\end{gathered}
$$

and

$$
N_{c}=\frac{1}{2}\left(N_{c c}+N_{v c}\right)
$$

Letting $\theta_{o \mid o}$ denote the conditional probability that a site bears an $o$ atom, given that a NN of this site does, we have

$$
N_{o o}=2 N_{o} \theta_{o \mid o}=2 N_{o} \frac{\theta_{o}}{\theta_{o}+\theta_{v}}
$$

where we used the fact that if a site bears $o$, then its NN cannot harbor $c$. By the same reasoning

$$
\begin{aligned}
& N_{o v}=2 N_{0} \frac{\theta_{v}}{\theta_{o}+\theta_{v}} \\
& N_{c c}=2 N_{c} \frac{\theta_{c}}{\theta_{c}+\theta_{v}}
\end{aligned}
$$


and

$$
N_{c v}=2 N_{c} \frac{\theta_{v}}{\theta_{c}+\theta_{v}}
$$

Combining Eqs. (11), (15) and (17) we then have

$$
N_{v v}=2 N_{v}\left[1-\frac{\theta_{o}}{\theta_{o}+\theta_{v}}-\frac{\theta_{c}}{\theta_{c}+\theta_{v}}\right]
$$

Note that in general $N_{v v}<2 N_{v} \theta_{v}$, the value it would have in the absence of the prohibition against $c-o$ NN pairs. In order to eliminate such pairs, vacancies must, as it were, be redistributed to create more $v-o$ and $v-c$ pairs than would be present under completely random mixing, and in this redistibution the number of $v-v$ pairs is diminshed.

Now using $\theta_{i j}=N_{i j} / 2 N$ and $\theta_{i \mid j}=\theta_{i j} / \theta_{j}$, we may proceed to assign rates to the various adsorption/reaction events in the model. Consider, for example, nonreactive adsorption of c. This occurs at an intrinsic rate of $Y$, and requires a vacant site whose four NNs are free of $o$. The probability that a site does not harbor $o$, given that its NN is vacant, is $1-\theta_{o \mid v}=\theta_{v} /\left(\theta_{o}+\theta_{v}\right)$, so that the rate $W_{1}$ (or expected number of events per site and unit time) of nonreactive $c$ adsorption is

$$
W_{1}=Y \theta_{v}\left(\frac{\theta_{v}}{\theta_{o}+\theta_{v}}\right)^{4} \equiv Y \theta_{v} \alpha
$$

The rates for the remaining events, listed in Table I, are found in the same manner (for further details see [2, 17]). We use the notation

$$
\theta_{v v}=\theta_{v}\left[1-\frac{\theta_{o}}{\theta_{o}+\theta_{v}}-\frac{\theta_{c}}{\theta_{c}+\theta_{v}}\right]
$$

and

$$
\gamma=\left(\frac{\theta_{v}}{\theta_{c}+\theta_{v}}\right)^{3}
$$

From the rates listed in Table I, one readily derives the following equations of motion for the coverages:

$$
\begin{aligned}
& \dot{\theta}_{o}=2 \bar{Y} \theta_{v v} \gamma-Y \theta_{v}(1-\alpha) \\
& \dot{\theta}_{c}=Y \theta_{v} \alpha-2 \bar{Y} \theta_{v v}(1-\gamma)
\end{aligned}
$$

Numerical integration of these equations reveals that for $Y<Y_{s}^{>}=0.56101$, starting from an empty lattice, the system attains an active steady state. $Y_{s}$ marks a spinodal point, 
the limit of stability of the active phase [7]. The site approximation does not reproduce the O-poisoning transition, yielding $Y_{s}^{<}=0$.

The present formulation is an improved version of the one-site approach presented in [2]. In the latter formulation one finds $Y_{s}^{>}=0.5615$, but, as noted, the final state (for $Y>Y_{s}^{>}$) has a small admixture of $o$ in the $c$-poisoned phase, in contradiction to the prohibition against $c-O$ NN pairs. The present formulation eliminates this flaw and provides a slightly improved estimate for $Y_{s}^{>}$.

In the preceding discussion we consider the thermodynamic limit (there is no reference to the system size). The model exhibits an active phase below the spinodal value $Y_{s}^{>}$. In finite systems it is not possible to avoid poisoning. But for large system sizes, we expect, for a range of $Y$, a long-lived metastable state, which we call the quasi-stationary state. Increasing the system size, the lifetime of the QS state goes to infinity, and its properties become equivalent to that of true active stationary state. In the next section we use the QS probability distribution in order to study finite systems.

\section{QUASI-STATIONARY PROBABILITY DISTRIBUTIONS}

The dynamics of a stochastic process such as the ZGB model is governed by a master equation (ME) for the probabilities in state space; the latter consists of all possible configurations of the system. Since the transition rates of the model do not satisfy detailed balance, one cannot determine the steady-state probability distribution without solving the ME. We may write a simplified ME, based on a cluster approximation, which, depending on the truncation level, includes certain fluctuations.

In the simplest case, one truncates the ME at the site level. This means that the state of the system is specified by the site occupation numbers $c, o$ and $v$, without regard to the relative positions of the adsorbed species on the surface. (Evidently, the fluctuations preserved at this level are those in the total occupancy.) Consider a square lattice of $N$ sites, so that $c+o+v=N$. The state of the ZGB model in the site approximation is given by the pair $(c, v)$; the model defines a Markov chain in the $c-v$ plane (see Fig. 1 ), with $\theta_{c}=c / N$, etc. The O-poisoned and CO-poisoned (absorbing) states are $(0,0)$ and $(N, 0)$, respectively.

In the site approximation, the probability distribution $P_{c, v}(t)$ follows the $\mathrm{ME}$ 


$$
\dot{P}_{c, v}(t)=\sum_{c^{\prime}, v^{\prime}} W_{\left(c^{\prime}, v^{\prime}\right) \rightarrow(c, v)} P_{c^{\prime}, v^{\prime}}(t)-P_{c, v}(t) \sum_{c^{\prime}, v^{\prime}} W_{(c, v) \rightarrow\left(c^{\prime}, v^{\prime}\right)}
$$

where $W_{(c, v) \rightarrow\left(c^{\prime}, v^{\prime}\right)}$ is the transition rate. In terms of the ZGB rates shown in Table 1, we have:

$$
\dot{P}_{c, v}=W_{1} P_{c-1, v+1}+W_{2} P_{c, v-1}+W_{3} P_{c, v+2}+W_{4} P_{c+1, v}+W_{5} P_{c+2, v-2}-\sum_{i=1}^{5} W_{i} P_{c, v}
$$

In Eq. (14) it is understood that each of the $W_{i}$ is to be evaluated for the same values as the associated probability factor (that is, in the first term, $W_{1}$ must be evaluated for $(c-1, v+1)$, etc. $)$.

"Poisoning" in the ZGB model corresponds to the system falling into one of the absorbing states (see Fig.1). This means that, for a finite system, the stationary probability distribution is nonzero only for absorbing configurations, that is,

$$
\lim _{t \rightarrow \infty} P_{c, v}(t)=p \delta_{(c, v),(0,0)}+(1-p) \delta_{(c, v),(N, 0)}
$$

(The weight $p$ depends on $N, Y$, and the initial distribution.)

We introduce the QSD via the hypothesis that, as $t \rightarrow \infty$, the probability distribution conditioned on survival attains a time-independent form. Then the QS probability distribution $Q_{c, v}$ is given by

$$
Q_{c, v}=\lim _{t \rightarrow \infty} P_{c, v}(t) / S(t)
$$

where $S(t)$ is the survival probability, i.e., the probability that the system has not fallen into the absorbing state up to time $t$. In the Appendix, we present results on the QS distribution for the ZGB model on a complete graph, which admits an exact description in terms of a single variable.

One method for generating the QSD is by integrating the master equation until the distribution $P_{c, v}^{s} \equiv P_{c, v}(t) / S(t)$, with $\left.S(t)=1-P_{0,0}(t)-P_{N, 0}(t)\right)$ attains a time-independent form. For moderately large systems $(N \geq 100)$, standard schemes such as the fourth-order Runge-Kutta method [19], require long integration times, because a small time step is needed to avoid numerical instabilities. An alternative method is available [20], based on writing the evolution in the form: 


$$
\dot{P}_{c, v}(t)=-W_{c, v} P_{c, v}(t)+A_{c, v}(t)
$$

where

$$
W_{c, v} \equiv \sum_{c^{\prime}, v^{\prime}} W_{(c, v) \rightarrow\left(c^{\prime}, v^{\prime}\right)}
$$

and

$$
A_{c, v}(t)=\sum_{v^{\prime} \geq 1, c^{\prime}} W_{\left(c^{\prime}, v^{\prime}\right) \rightarrow(c, v)} p_{c^{\prime}, v^{\prime}}(t)
$$

Suppose the probability distribution has attained a QS form at some time $t$. Using normalization, $\sum_{v \geq 1, c} Q_{c, v}(t)=1$ and Eq. (17), we have

$$
A_{c, v}=\left(W_{c, v}-A_{0}\right) P_{c, v}
$$

where

$$
A_{0} \equiv \sum_{v^{\prime}>1, c^{\prime}} W_{\left(c^{\prime}, v^{\prime}\right) \rightarrow(c, 0)} P_{c, v}=-\sum_{v \geq 1, c} \dot{P}_{c, v}(t)
$$

is the decay rate of the survival probability.

For the ME, Eq. (12), $W_{c, v}=\sum_{i=1}^{5} W_{i}$ and

$$
A_{c, v}=W_{1} P_{c-1, v+1}+W_{2} P_{c, v-1}+W_{3} P_{c, v+2}+W_{4} P_{c+1, v}+W_{5} P_{c+2, v-2}
$$

The decay rate is $A_{0}=W_{1} P_{N-1,1}+W_{3} P_{0,2}$. In the quasi-stationary regime we have

$$
Q_{c, v}=\frac{A_{c, v}}{W_{c, v}-A_{0}} \quad(v \geq 1)
$$

which suggests the following iteration scheme:

$$
P_{c, v}^{\prime}=a P_{c, v}+(1-a) \frac{A_{c, v}}{W_{c, v}-A_{0}}
$$

where $a$ is a parameter and $A_{c, v}$ is evaluated using the distribution $P_{c, v}(t)$ The new distribution $P_{c, v}^{\prime}$ must be normalized after each iteration. In this way, one can construct the QS state from any normalized initial distribution $P_{c, v}(t)$. This iterative method exhibits good convergence for $a=0.5$. 


\section{RESULTS}

We construct the QS distributions for the ZGB model at the one-site level, via both the Runge-Kutta method and the iterative scheme, which as expected yield the same QS distribution. Using $a=0.5$, we find that the iterative method converges to the QS distribution about 100 times faster than via the RK method using the same precision. This gain in efficiency is associated with the small time step required to maintain numerical stability in the RK scheme. In Fig. 2, we show a typical QS distribution, $Q_{c, v}$, for the ZGB model near the spinodal point. It is clearly bimodal, with one maximum at $c=N-1$, near the CO-poisoned state, and another, broader one that corresponds the active state.

Figure 3 shows the time evolution of the coverages (conditioned on survival), for two different initial conditions, and $Y$ fixed at the spinodal value $Y_{s}^{>}$obtained from the $\mathrm{MF}$ theory of Sec. II. It is clear that, although the relaxation to the QS values is very different in the two cases, both reach reach the same QS coverages. The relaxations to the QS state for $Y<Y_{s}^{>}$and $Y>Y_{s}^{>}$are compared in Fig. 4 (Since the iterative method provides only the QS distributions, the evolution is calculated via the Runge-Kutta method in this case.)

It is also possible to discuss QS properties for $Y>Y_{s}$, where an active stationary state does not exist, even in the thermodynamic limit. This is a finite-size effect, analogous to a nonzero magnetization in the Ising model above the critical temperature, on a finite lattice. This effect was also reported for the contact process on a complete graph [13].

The marginal QS distribution $Q_{c}$ (Fig. 5) becomes bimodal as $Y \rightarrow Y_{s}^{>}$, consistent with a discontinuous phase transition and the emergence of a hysteresis loop when $N \rightarrow \infty$. The lifetime $\tau$ of the QS state is given by

$$
\tau=1 / A_{0}
$$

As seen in Fig. 6, $\tau$ becomes very large as we increase $N$, for $Y<Y_{s}^{>}$. The lifetime increases exponentially with $N$ (Fig. 6 - inset), as found for the $\mathrm{CO}$ poisoning time on finite lattices 21].

Fig. 7 shows the mean number of adsorbed CO molecules, $\langle c\rangle$, and of vacant sites $\langle v\rangle$, in the QS state. It also displays the QS moment ratios $m_{c}=\left\langle C^{2}\right\rangle /\langle C\rangle^{2}$ and $m_{v}=\left\langle V^{2}\right\rangle /\langle V\rangle^{2}$ as functions of $Y$. The quantity $m$ is analogous to Binder's reduced fourth cumulant 22] at an equilibrium critical point. At a continuous phase transition to an absorbing state, 
we expect $m$ for the order parameter to attain a universal, size-independent value, due to scaling of the probability distribution [24, 25]. In the present case of a discontinuous phase transition, $m_{c}$ and $m_{v}$ can be expected to grow due to the bimodal nature of the probability distribution. Very large values of $m_{v}$ can be attained as the coverge $\theta_{v}$ tends to its minimum, $1 / N$. For the finite systems studied here, the peaks of $m_{c}$ and $m_{v}$ do not fall at the same values of $Y$, as would be expected for an infinite system. The positions of the maxima appear to approach one another as $N \rightarrow \infty$; an extrapolation to $N \rightarrow \infty$ confirms the peak values occur at $Y_{s}^{>}$as given by mean-field theory.

\section{A. Noninstantaneous reactions}

One of many possible extensions for the ZGB model that have been proposed relaxes the condition of instantaneous reactions between adsorbed $\mathrm{O}$ and $\mathrm{CO}$. [4]. The key difference between this NI model and the ZGB model is that there is no longer any prohibition against $c-o$ NN pairs. There is now a finite mean reaction time for $\mathrm{O}$ and $\mathrm{CO}$ adsorbed at NN sites to form $\mathrm{CO}_{2}$; the reciprocal of the reaction time is the reaction rate $R$. (We continue to assume instantaneous desorption of $\mathrm{CO}_{2}$.) The transitions are listed in Table II; rates are obtained as in Table I. (The prefactor 4 in rate $W_{3}$ is the coordination number of the square lattice.)

In this case, the mean-field equations are:

$$
\begin{aligned}
& \dot{\theta}_{o}=2(1-Y) \theta_{v}{ }^{2}-4 R \theta_{o} \theta_{c} \\
& \dot{\theta}_{c}=Y \theta_{v}-4 R \theta_{o} \theta_{c}
\end{aligned}
$$

A simple analysis of equation (26) shows that $Y_{s}^{>} \sim \sqrt{2 R}$ as $R \rightarrow 0$, grows monotonically with $R$, attaining the limiting value $Y_{s}^{>}=2 / 3$ as $R \rightarrow \infty$. This value is different from the spinodal found in the site-approximation for the ZGB model, which involves instantaneous reactions. Since $\lim _{R \rightarrow \infty} Y_{s}^{>} \neq Y_{s, Z G B}^{>}$, the limit of infinite reaction rate is singular. The singular nature of this limit derives from the fact that the instantaneous reaction condition imposes certain constraints on configurations, thereby altering the algebraic form of the mean-field equations (a more extensive discussion may be found in [4]). In particular, for $R \rightarrow \infty, \theta_{o}$ and $\theta_{c}$ cannot both be nonzero, whereas both coverages are nonzero in the active phase of the ZGB model. 
The probability distribution for the NI model follows the master equation:

$$
\dot{P}_{c, v}(t)=R_{1} P_{c-1, v+1}+R_{2} P_{c, v+1}+R_{3}\left[P_{c+1, v-1}+P_{c, v-1}\right]-\left[R_{1}+R_{2}+2 R_{3}\right] P_{c, v}(t) .
$$

We turn now to a discussion of the QS properties, obtained using the iterative method based on Eq. (23). The lifetime of the QS state, in the reactive window, increases with $R$ (see Fig. 8). The displacement of the maximum position of $\tau$ (as a function of $Y$ ) parallels the variation in $Y_{s}^{>}(R)$. The maximum lifetime (i.e., varying $Y$ ) tends to a finite value, different from that from the original ZGB model, when $R \rightarrow \infty$. This is because the flux to the absorbing configurations, which determines the lifetime of the QS state, and which, in the site approximation, is given by $A_{0}=W_{1} Q_{N-1,1}+W_{3} Q_{0,2}$, is reduced as $R$ increases, since the latter reduces the QS probabilities $Q_{N-1,1}$ and $Q_{0,2}$.

Figs. 9 and 10 show the QS coverages $\theta_{c}$ and $\theta_{v}$. As $R$ increases the transition becomes progressively "harder," i.e., the coverage $\theta_{c}$ just below the transition approaches zero while that just above approaches unity. In the limit $R \rightarrow \infty$, the $c$ coverage is a step-function: $\theta_{c}=\Theta\left(Y-Y_{s}^{>}\right)$. As before, the QS coverages tend to the macroscopic curves (furnished by mean-field theory) when $N \rightarrow \infty$. Due to the low $c$ coverage in the active phase for large $R$, the moment ratios $m_{c}$ differ strongly from those of the ZGB model. But as always, the peak in $m_{c}$ tends to the spinodal as $N \rightarrow \infty$ (see Fig. 11).

\section{SUMMARY}

We study the quasi-stationary properties of the ZGB model for the catalytic oxydation of $\mathrm{CO}$, and of a related model with noninstantaneous reactions. The QS distribution is constructed numerically via a bivariate extension of the iterative method [20]. For the simple case of a complete graph, we obtain exact (numerical) results for QS properties. Although our analysis of the models with NN reactions on a square lattice is based on site-approximations, which cannot capture critical fluctuations, the approach allows one to put some fluctuations "back into" mean-field theory, specifically, fluctuations in the overall coverages, since we assume spatial homogeneity. This enables us to investigate finite-size effects and moment ratios that are out of reach with other approaches. We derive a revised site approximation for the ZGB model that respects the prohibition against $c-o$ NN pairs,

eliminating the inconsistency noted in previous formulations [2, 3, 4], while providing a 
reasonably good estimate of the spinodal point.

The present study confirms two fundamental properties of the ZGB and NI models: (1) the discontinuous phase transition is signaled by a bimodal QS probability distribution; (2) the lifetime of the QS state grows exponentially with system size, in the reactive phase. The latter implies that the system will typically spend a long time in the QS state, following a relatively brief transient, except near the spinodal, where relaxation slows down. We have also noted sharply increased values of the moment ratios $m_{c}$ and $m_{v}$ in the vicinity of the transition. Since the $\mathrm{CO}_{2}$ production rate is closely related to $\theta_{v}$, one should expect to observe large fluctuations in product concentrations in the gas phase, as the spinodal is approached. One may envision using these fluctuations as part of a control scheme that adjusts the $\mathrm{CO}$ partial pressure (represented by the parameter $Y$ in the models), to optimize production while avoiding poisoning.

An interesting result is that at the level of mean-field theory, the ZGB model is not equivalent to the infinite-reaction-rate limit of the model with noninstantaneous reactions. The ZGB model is characterized by the constraint that any $c-o$ NN pair react before any further adsorption events, a condition that is also realized in the NI model, for sufficiently large $R$. Mean-field theory is however incapable of representing this situation, since, in the absence of a prohibition against $c$ - $O$ pairs, the NI model in the $R \rightarrow \infty$ limit simply prohibits simultaneous nonzero coverages of $\mathrm{O}$ and CO. Since truly instantaneous reactions do not exist, one might suppose that the NI model, with an appropriately large rate $R$, provides the correct description of fast (but not instantaneous) surface reactions. In meanfield approximation however, the large- $R$ limit of the NI model appears less realistic than the ZGB model, which permits simultaneous presence of adsorbed $\mathrm{O}$ and $\mathrm{CO}$. In this sense, the choice of model in the description of cooperative kinetics should involve the approximation that one intends to use, not only the intrinsic nature of the model.

QS analysis promisses to be a useful technique in the study of catalysis models on nanome-

ter scales [9]. Possible directions for future work include the study of metastability via QS distributions, and Monte Carlo simulations of the QS state, in catalytic models and other processes exhibiting absorbing states.

\section{ACKNOWLEDGMENT}

This work was supported by CNPq and CAPES, Brazil. 


\section{APPENDIX: ZGB MODEL ON A COMPLETE GRAPH}

A complete graph is one in which all sites are neighbors. When formulated on such a structure, a stochastic lattice model defines a stochastic process defined by one or a few variables, thereby permitting an exact analysis, as in the case of the contact process 13]. Since each site interacts with all others, the behavior is mean-field-like. Due to the prohibition against $c-o$ NN pairs, in the ZGB model on a complete graph, only one species ( $c$ or $o$ ) may be present at any moment. Letting $n=c-o$, we can describe a system of $N$ sites completely by a single variable, with $n=-N$ representing the O-poisoned state and $n=N$ the CO-poisoned state. The number of vacant sites is $v=N-|n|$.

Each adsorption event leads either to $n \rightarrow n+1$ or to $n \rightarrow n-2$. The transition rates are

$$
W_{n \rightarrow n+1}=Y\left(1-\frac{|n|}{N}\right)
$$

and

$$
W_{n \rightarrow n-2}=\bar{Y}\left(1-\frac{|n|}{N}\right)\left(1-\frac{|n|+1}{N}\right) .
$$

The process obeys the master equation:

$$
\begin{aligned}
\dot{P}_{n}(t)= & Y u_{n-1} P_{n-1}(t)+\bar{Y} u_{n+2}\left(u_{n+2}-\frac{1}{N}\right) P_{n+2}(t) \\
& -u_{n}\left[Y+\bar{Y}\left(u_{n}-\frac{1}{N}\right)\right] P_{n}(t)
\end{aligned}
$$

where $u_{n}=\left(1-\frac{|n|}{N}\right)$. The macroscopic equation for this process [21] is given by:

$$
\dot{\phi}(t)=Y(1-|\phi(t)|)-2 \bar{Y}(1-|\phi(t)|)^{2} .
$$

where $\lim _{N \rightarrow \infty} \phi(t)=n(t) / N$. In this limit, the system poisons with CO when $Y \geq 2 / 3$. For $Y<2 / 3$ there is a nontrivial fixed point at $\phi^{*}=1-(1-Y) / 2 Y$ witch is the attractor if $\phi(0)>-\phi *$. The point $\phi^{*}$ is the reactive stationary state for an infinite system.

Analyzing the master equation via the iterative method described in Sec. III, we obtain the QSD shown in Fig. 12. The QSD changes qualitatively as $Y$ approaches the spinodal. For small $Y$, the distribution has a single maximum near $\phi^{*}$. For $Y \simeq 2 / 3$ the distribution becomes bimodal, with one peak near $\phi^{*}$, related to the active phase, and another at $n=N-$ 1, related to the CO-poisoned state. Fig. 13 shows the QS activity for this model; as $N \rightarrow \infty$, it tends to the macroscopic limit (dashed line). As verified for the site approximation, the 
lifetime grows exponentially with $N$ in the active region $(0<Y<2 / 3)$ and linearly in the nonreactive region.

[1] R. M. Ziff, E. Gulari, and Y. Barshad, Phys. Rev. Lett., 56, 2553 (1986).

[2] R. Dickman, Phys. Rev. A, 34, 4246 (1986).

[3] P. Fischer and U. M. Titulaer, Surf. Sci., 221, 409-426 (1989).

[4] M. Dumont et al., J. Catalysis, 122, 95 (1990).

[5] J. W. Evans, Langmuir 7, 2514 (1991).

[6] V. P. Zhdanov and B. Kasemo, Surf. Sci. Rep. 20, 111 (1994).

[7] J. Marro and R. Dickman, Nonequilibrium Phase Transitions in Lattice Models (Cambridge University Press, Cambridge, 1999).

[8] T. Engel and G. Ertl, Advances in Catalysis v. 8 (Academic Press, New York, 1979).

[9] Y. Suchorski et al., Phys. Rev. Lett. , 82, 1907(1999);

Y. Suchorski et al., Phys. Rev. B, 63, 165417 (2001).

[10] A. M. Yaglom, Dokl. Akad. Nauk. SSSR 56, 797 (1947).

[11] P. A. Ferrari et al., Ann. Prob. 23, 501 (1995);

P. A. Ferrari, H. Kesten and A. Martínez, Ann. Appl. Prob. 6, 577 (1996).

[12] I. Nàsell, J. Theor. Biol. 211, 11 (2001).

[13] R. Dickman and R. Vidigal, J. Phys. A: Math. Gen., 35 1147, (2002).

[14] A.P.F. Atman and R. Dickman Phys. Rev. E, 66, 046135 (2002).

[15] F. Schlögol, Z. Phys., 253, 147 (1972).

[16] P. Grassberger, Z. Phys. B, 47, 465 (1982).

[17] D. ben-Avraham and J. Köler, Phys. Rev. A., 45, 8358 (1992).

[18] J. W. Evans and T. R. Ray, Phys. Rev. E,50, 4302 (1994).

[19] W. H. Press et al., Numerical Recipes (Cambridge University Press, Cambridge, 1996).

[20] R. Dickman, Phys. Rev. E, 65, 047701(2002).

[21] D. ben-Avraham et al., J. Phys. A: Math. Gen., 23, L613 (1990).

[22] K. Binder, Phys. Rev. Lett. , 47, 693(1981).

[23] V. P. Zhdanov and B. Kasemo, Surf. Sci. , 496, 251(2002).

[24] R. Dickman and J. Kamphorst Leal da Silva, Phys Rev. E58, 4266 (1998). 
[25] R. Dickman and M. A. Muñoz, Phys. Rev. E 62, 7632 (2000). 
TABLE I: Site mean-field theory for the ZGB model

\begin{tabular}{lll}
\hline \hline Event & Rate & $(c, v)$ to \\
\hline$v+c \downarrow \rightarrow c$ & $W_{1}=Y \theta_{v} \alpha$ & $(c+1, v-1)$ \\
$v+c \downarrow \rightarrow v$ & $W_{2}=Y \theta_{v}(1-\alpha)$ & $(c, v+1)$ \\
$v v+o o \downarrow \rightarrow o o$ & $W_{3}=\bar{Y} \theta_{v v} \gamma^{2}$ & $(c, v-2)$ \\
$v v+o o \downarrow \rightarrow v o$ & $W_{4}=2 \bar{Y} \theta_{v v} \gamma(1-\gamma)$ & $(c-1, v)$ \\
$v v+o o \downarrow \rightarrow v v$ & $W_{5}=\bar{Y} \theta_{v v}(1-\gamma)^{2}$ & $(c-2, v+2)$ \\
\hline \hline
\end{tabular}

TABLE II: Site mean-field theory for the finite reaction-rate model

\begin{tabular}{cll}
\hline \hline Event Rate & $(c, v)$ to \\
\hline$c \downarrow$ & $W_{1}=\theta_{v} Y$ & $(c+1, v-1)$ \\
$o o \downarrow$ & $W_{2}=2 \theta_{v}^{2} \bar{Y}$ & $(c, v-2)$ \\
$o c \uparrow$ & $W_{3}=4 R \theta_{c} \theta_{o}$ & $(c-1, v+2)$ \\
\hline \hline
\end{tabular}

TABLES 


\section{FIGURE CAPTIONS}

FIG. 1. Transitions in the ZGB model (site approximation). Circles denote absorbing states.

FIG. 2. A typical bimodal QS distribution $Q_{s}(c, v)$ near the spinodal point $(Y=0.56101)$.

FIG. 3. Time evolution of the mean values conditioned on survival for $Y=0.56101$ and system size $N=64$. Initial conditions, from top to bottom: half $c$ and half $v$; full $v$; half $o$ and half $v$. Dashed lines show the respective unconditioned values.

FIG.4.Relaxation for the QS mean values of $c$ for $Y=0.50, Y=0.56101$ and $Y=0.60$, from bottom to top. $N=64$. Dashed lines: unconditioned mean values

FIG. 5. Marginal distribution $Q_{c}$ for $\mathrm{Y}=.54, .55, .56, .57, .58$.

FIG. 6. Lifetime $\tau$ of the QS state as function of $\mathrm{CO}$ arrival rate $Y$, for the ZGB model. System sizes $N=36,64,100,256,400,512$ from bottom to top. Inset: Lifetime as function of the system size $N$ for $Y=0.50$.

FIG. 7. Mean values conditioned on survival $\langle c\rangle,\langle v\rangle$, and respective moment ratios, $m_{c}$ and $m_{v}$ for the original ZGB model without desorption. System size $N=$ $16,36,64,100,256,400,700$.

FIG. 8. Lifetime $\tau$ of the QS state for the NI model. System sizes $N=100$ (lower solid lines); $N=256$ (dashed lines); $N=400$ (upper solid lines). Reaction rates $R=0.1,1,10$, and $10^{6}$ from left to right. The dashed line is the lifetime for the ZGB model. Inset: detail of the same graph with $N=100$, showing the lifetime for the ZGB model (dashed line).

FIG. 9. QS coverage $\theta_{c}$ for the NI model; reaction rates as in Fig. 8. System sizes $N=100$ (solid lines) and $N=400$ (dashed lines).

FIG. 10. QS vacancy fraction $\theta_{v}$ for the NI model, as in Fig. 9.

FIG.11. Moment ratio $m_{c}$ for NI model. Reaction rates and system sizes as per Fig. 9.

FIG. 12. QSD for the ZGB model on a complete graph of 100 sites. 
FIG. 13. Quasistationary activity for the ZGB on a complete graph. The dashed line represents the macroscopic limit, Eq. (31). 


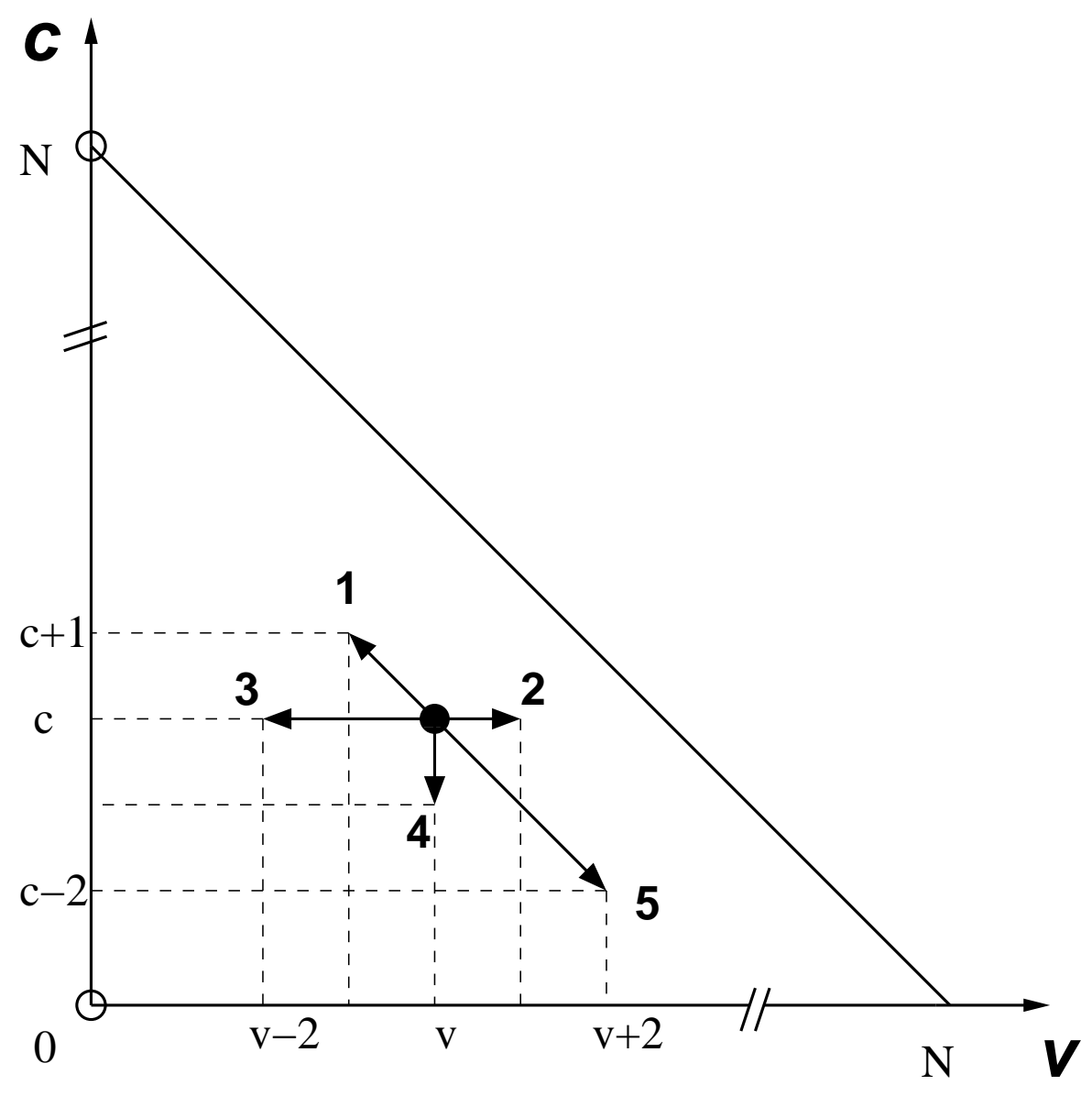









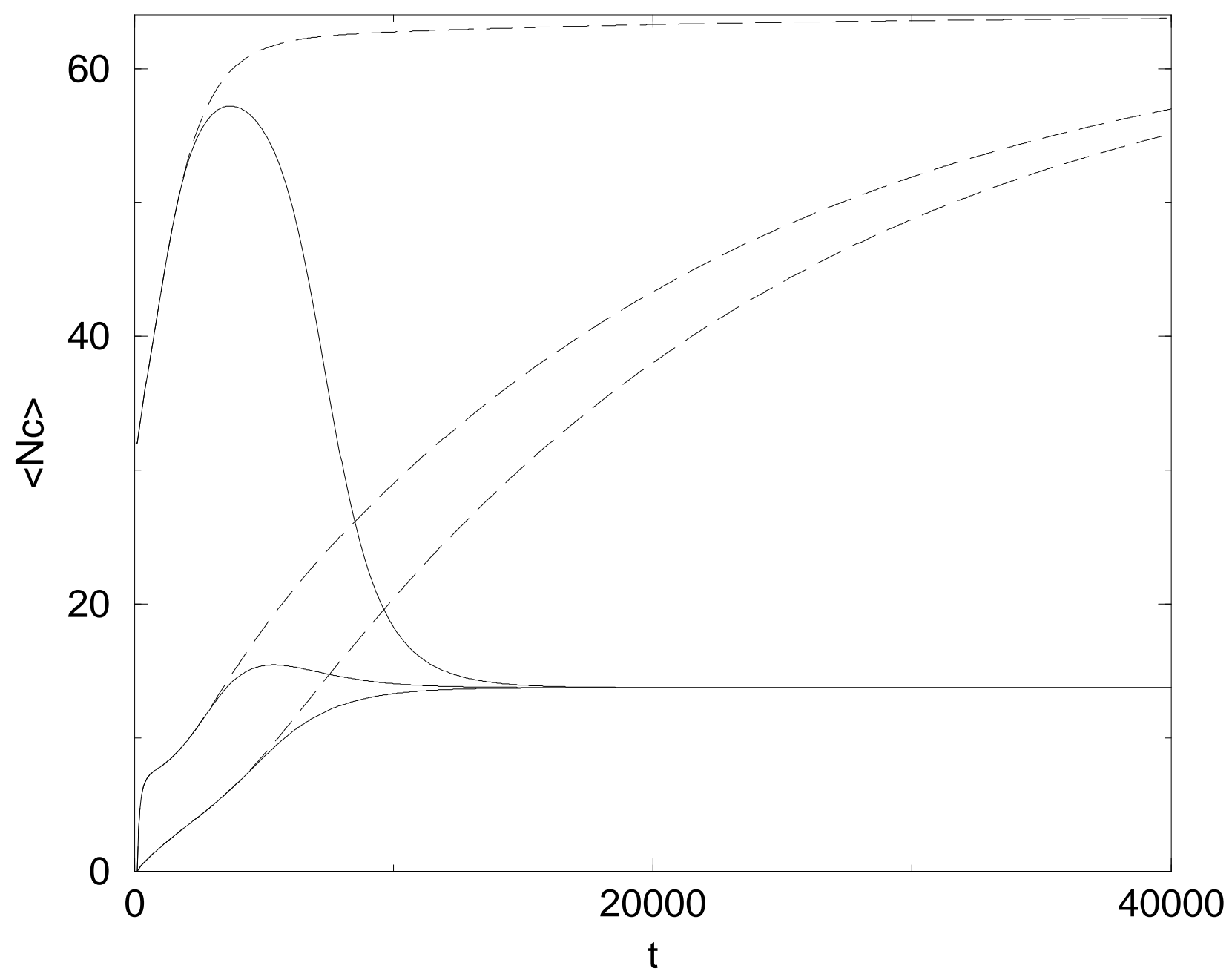




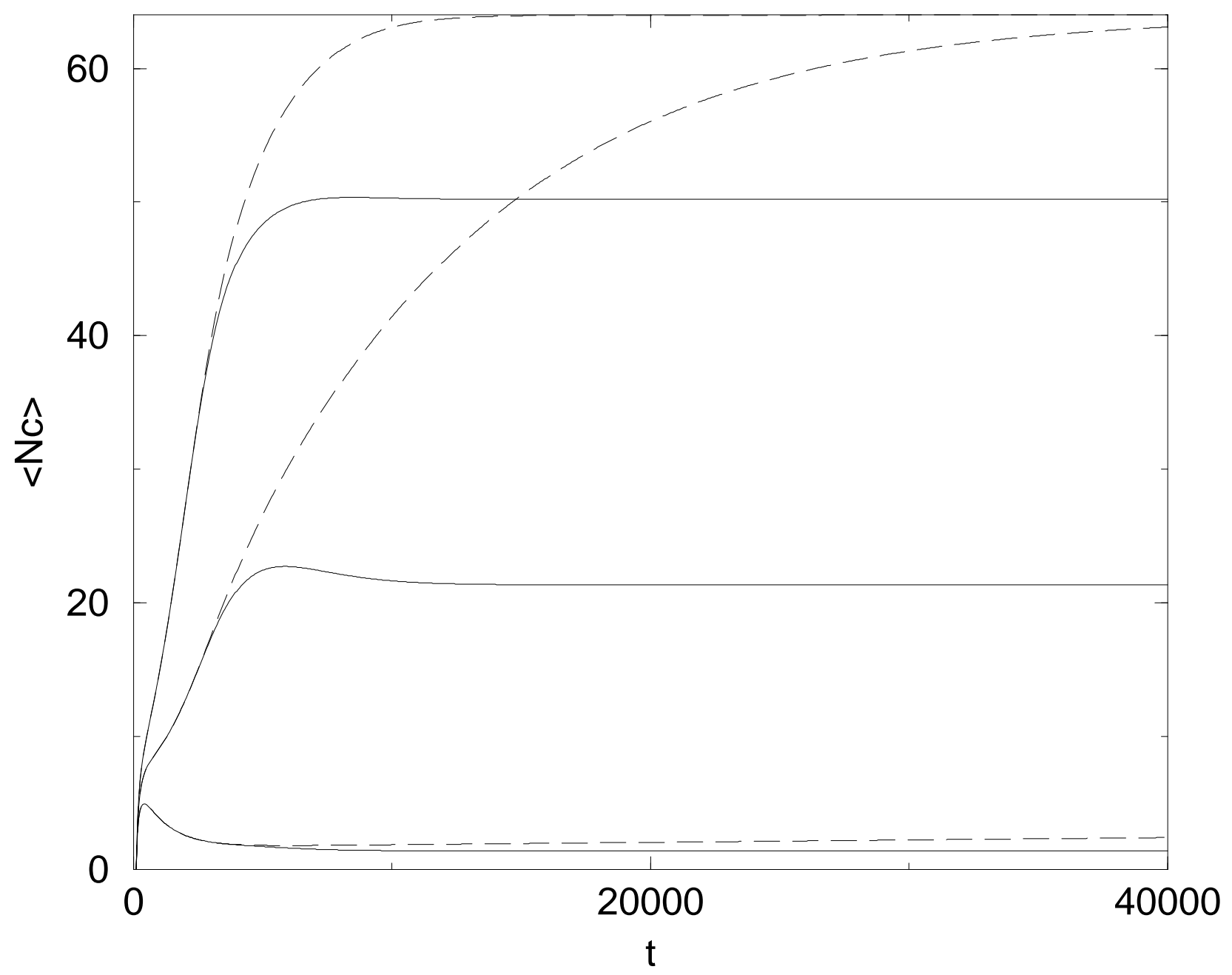




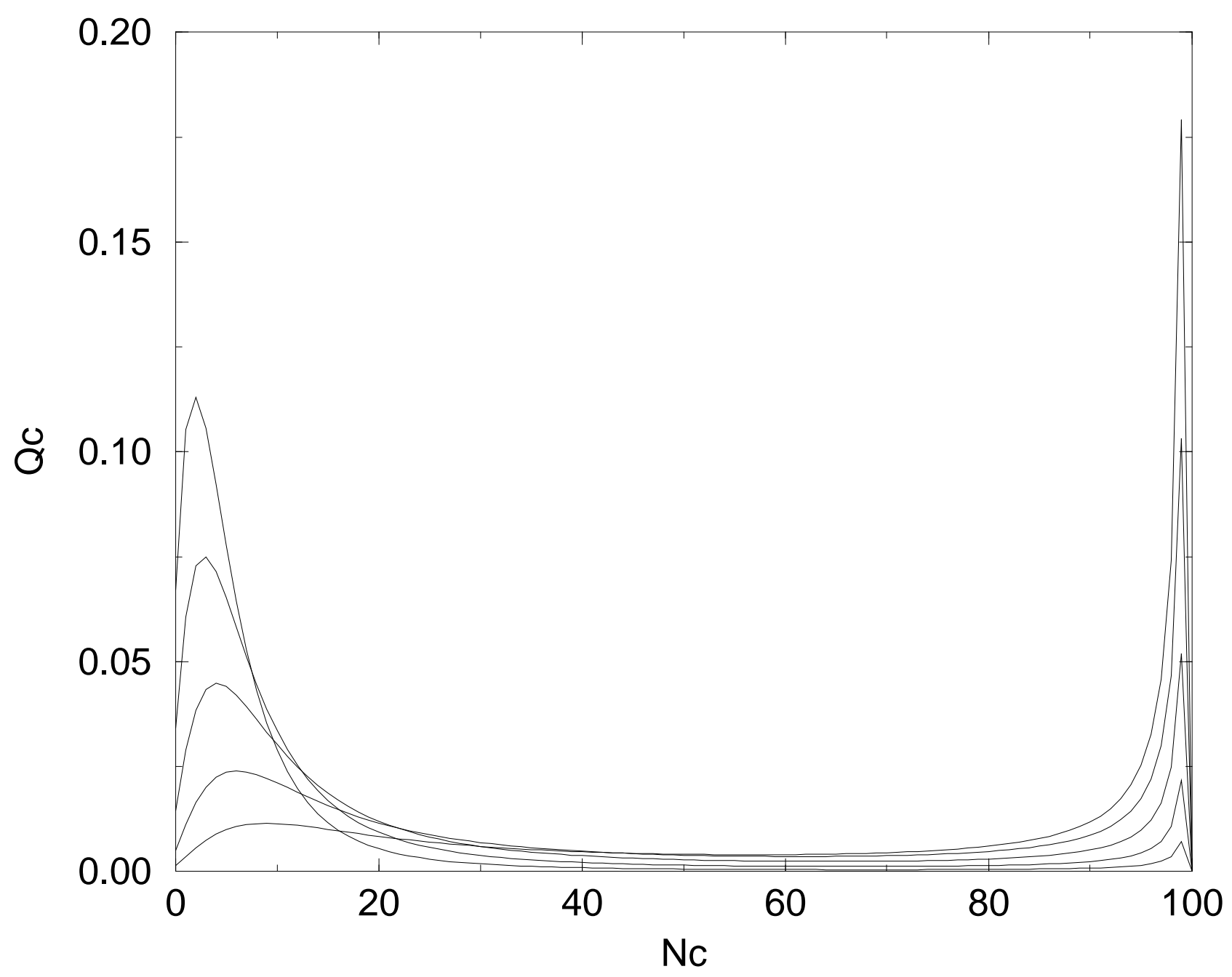




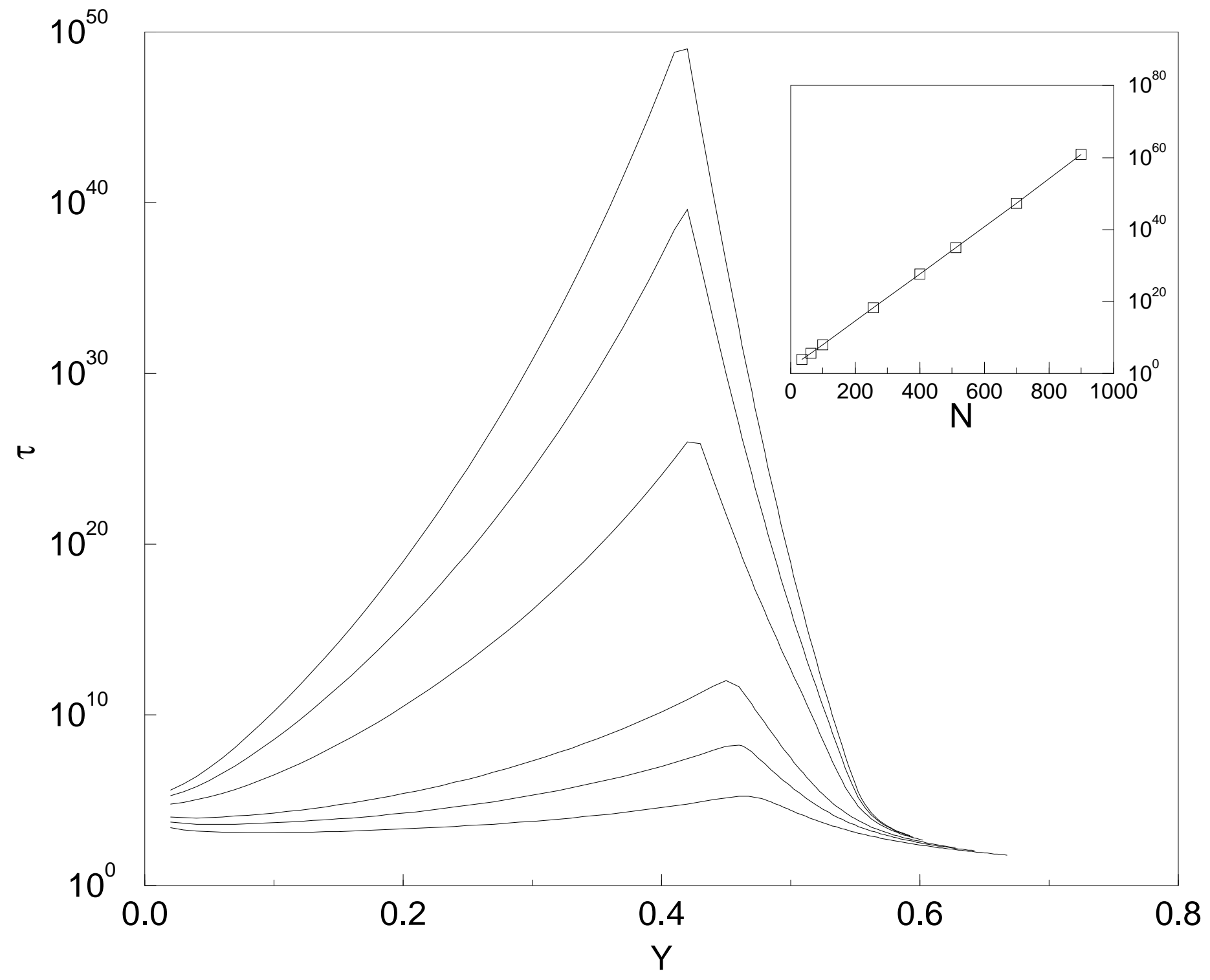



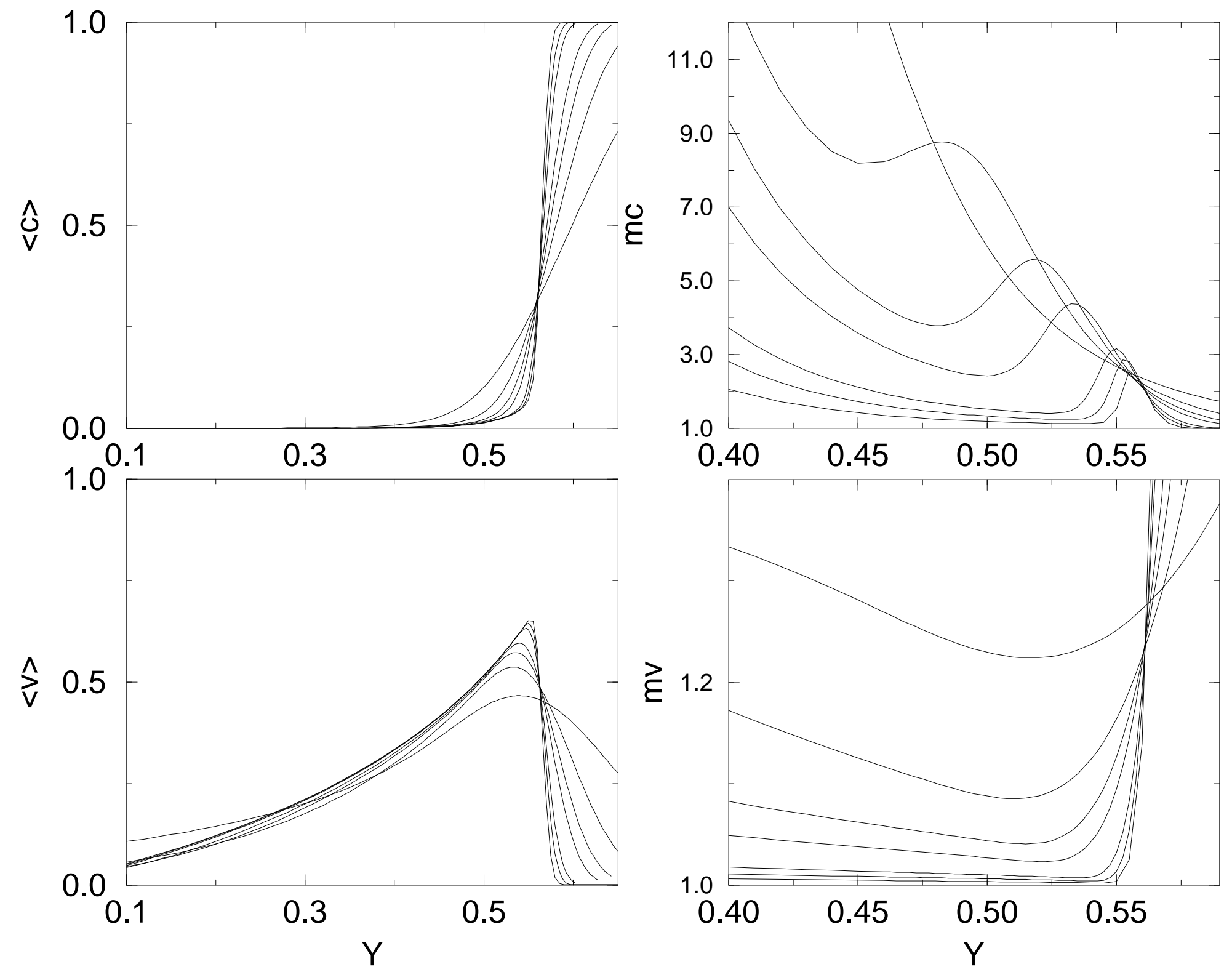


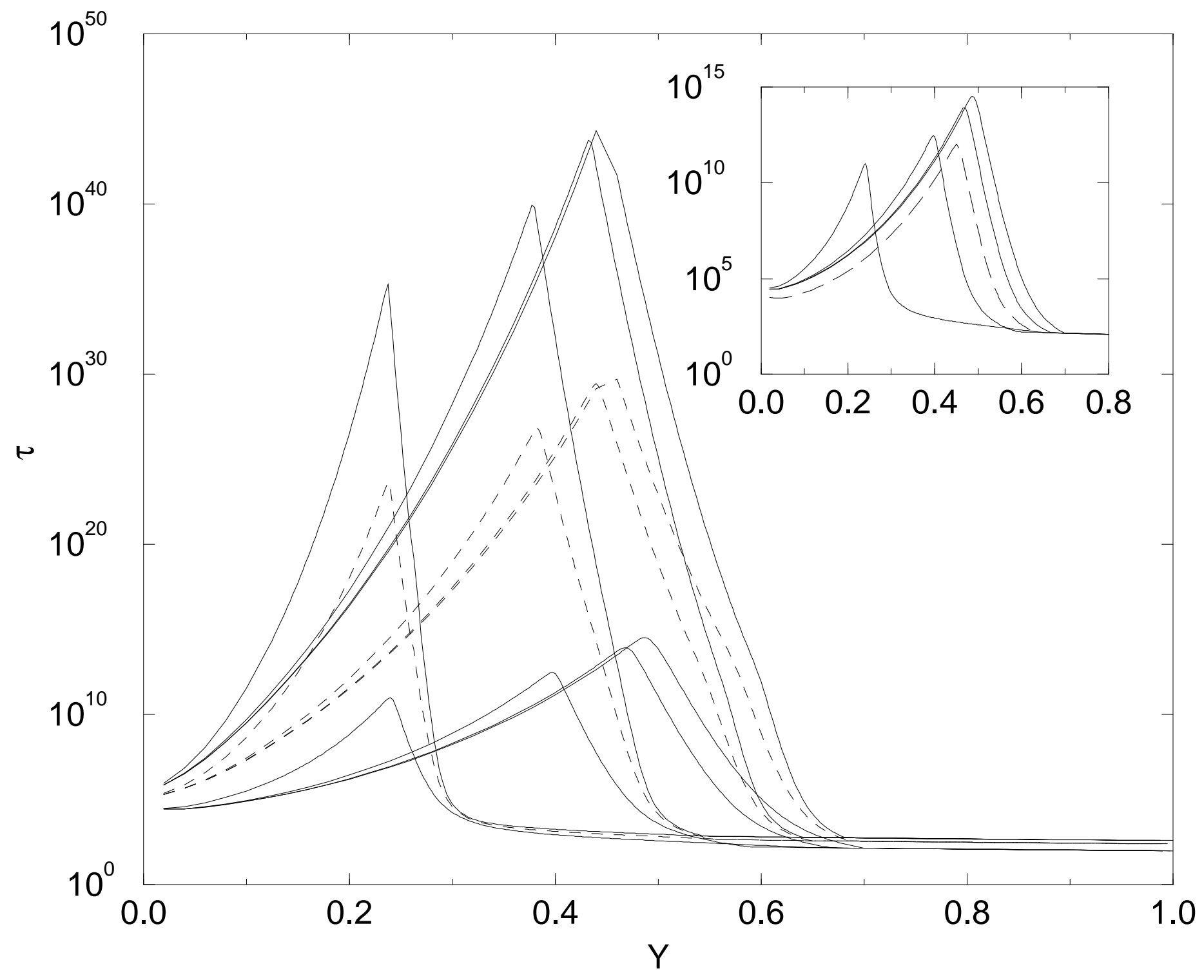




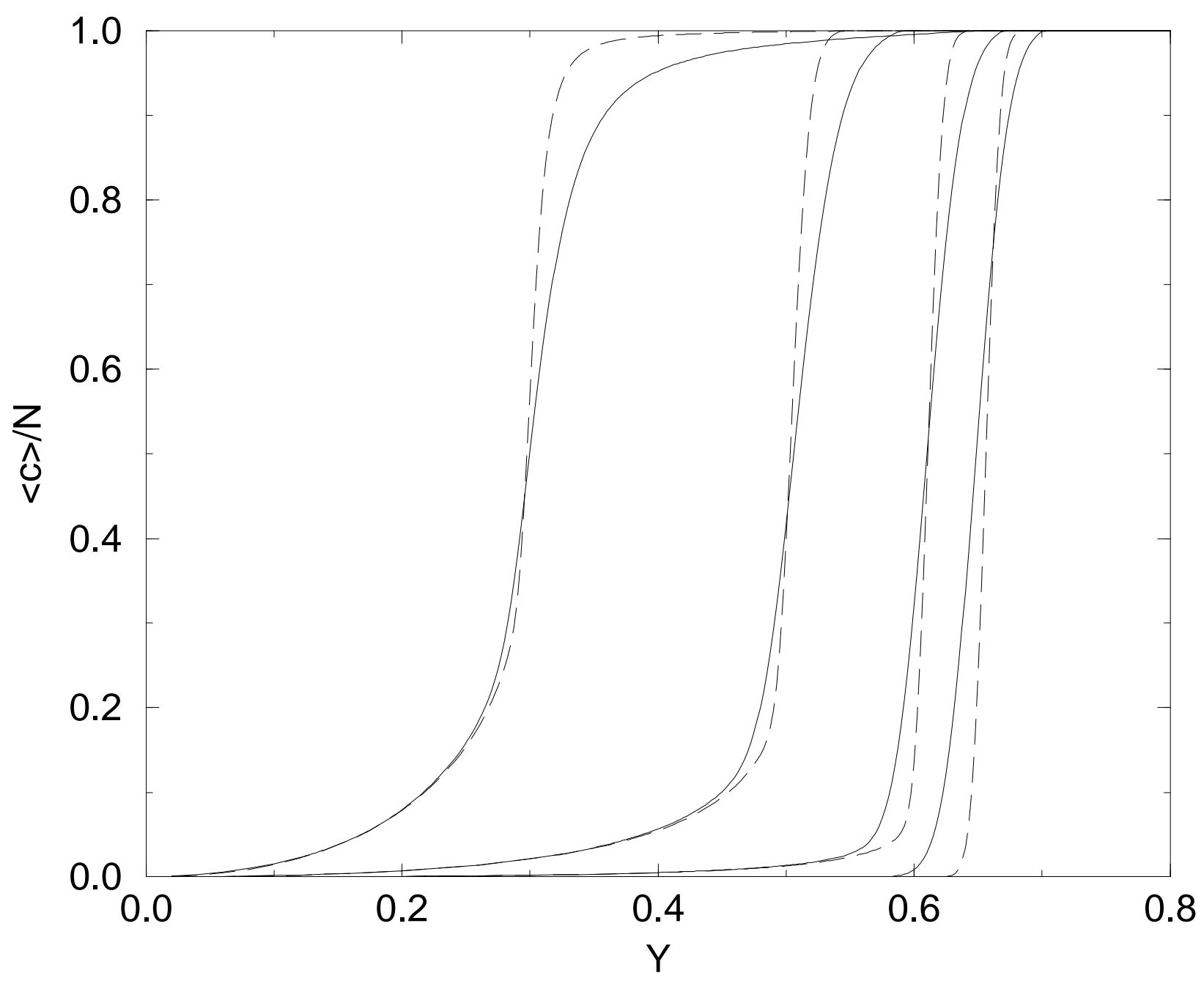




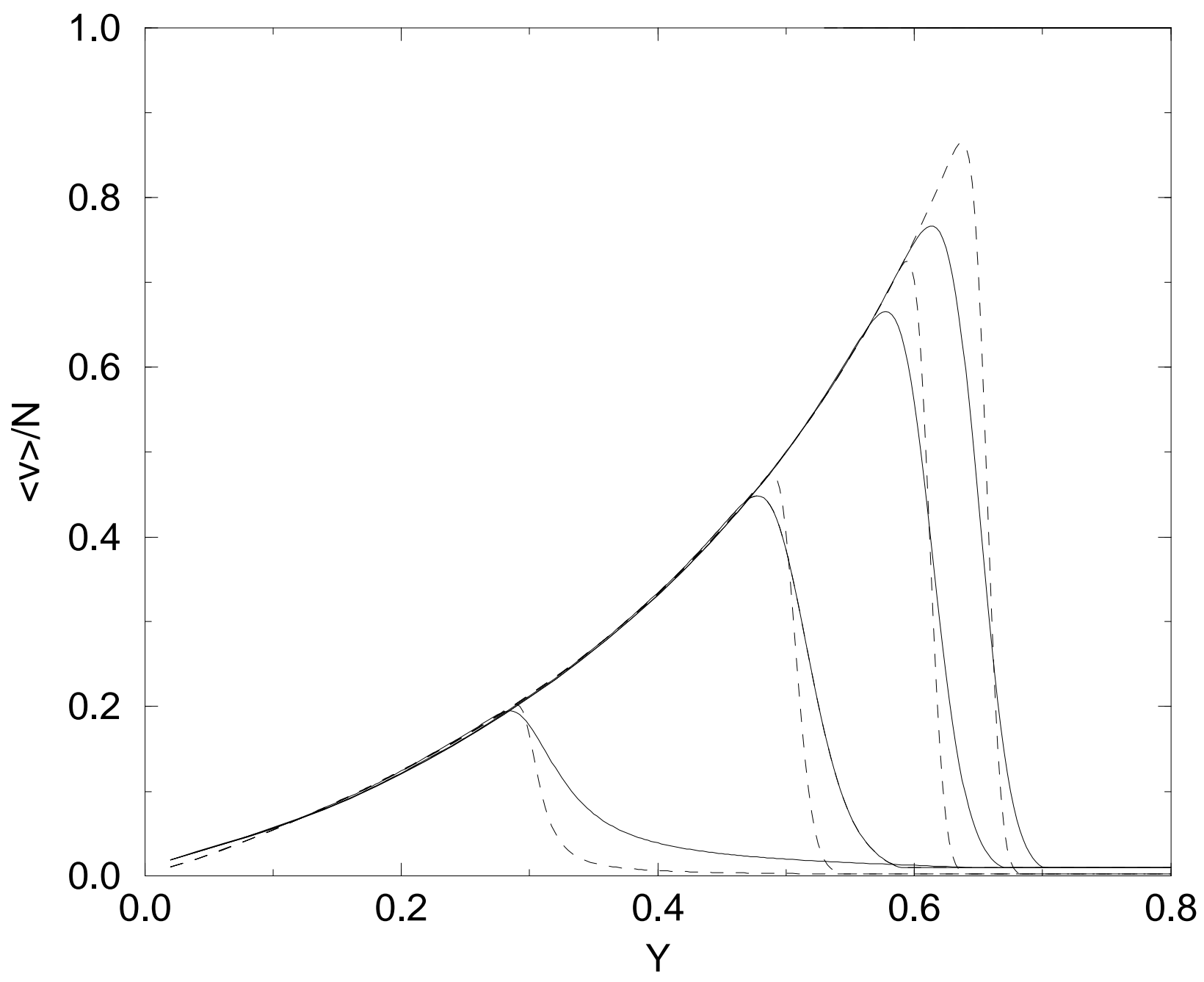




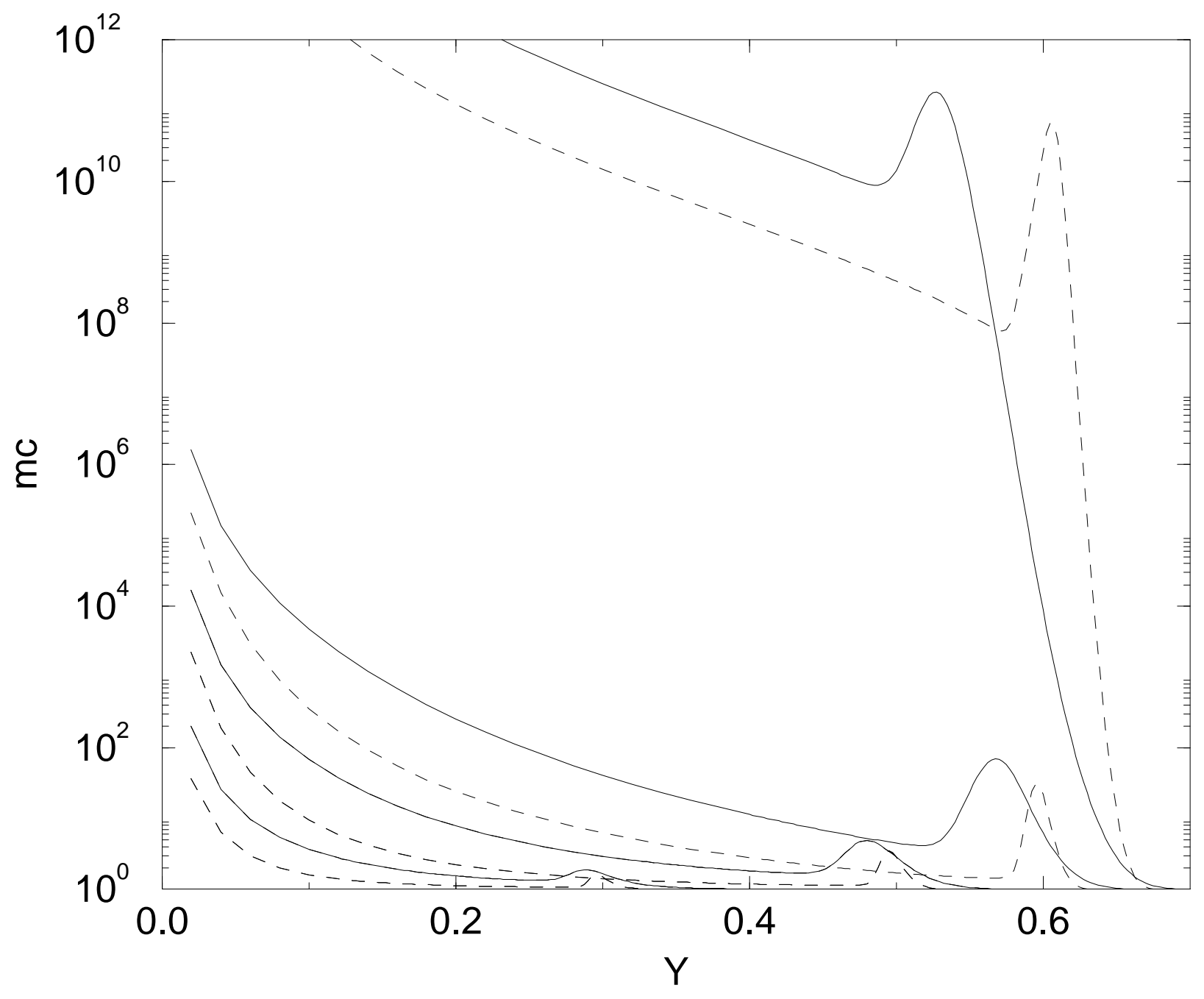




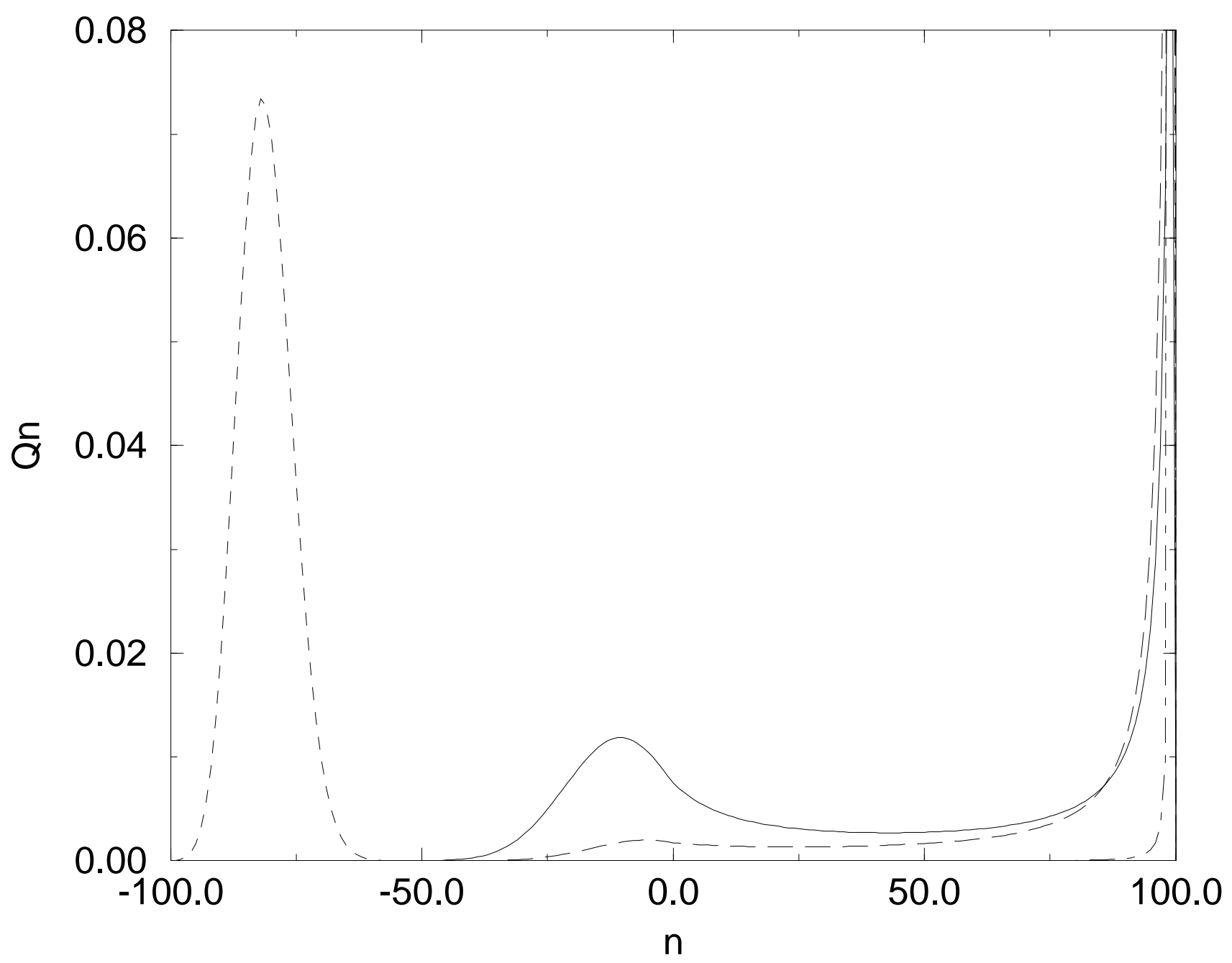




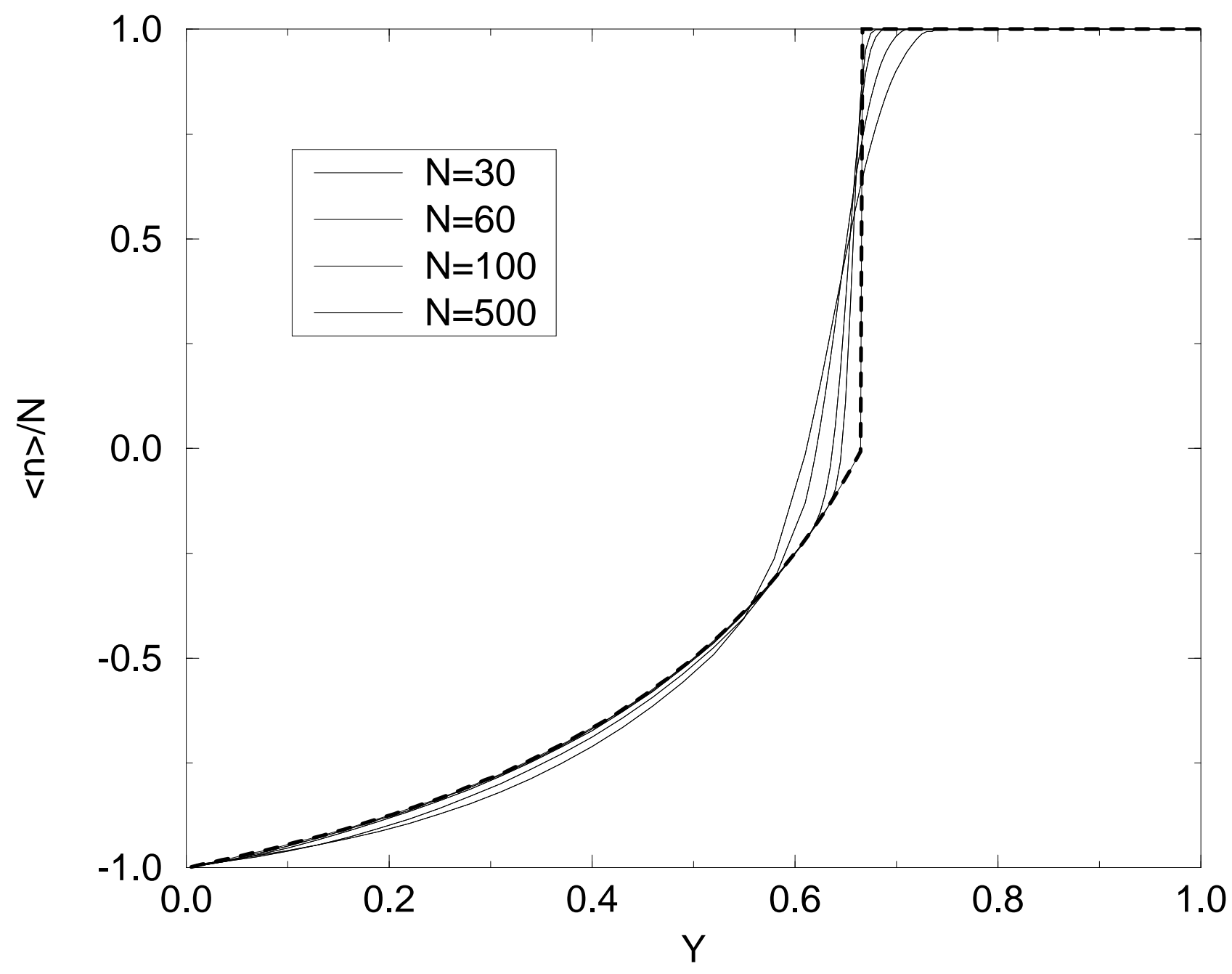

